\title{
ApARATOS DE AVANCE MANDibUlAR: ¿Mito O REALIDAD?
}

\author{
Natalia Parra Quintero, Res. Pos. Ortod. ${ }_{1}$ Paola María Botero Mariaca*, Ortodon. \\ ${ }_{1}$ Universidad Cooperativa de Colombia, sede Medellín, Colombia
}

Recibido: 26 de octubre del 2012 Aprobado: 17 de noviembre del 2012

* Autor de contacto: Paola María Botero M., Facultad de Odontología, Universidad Cooperativa de Colombia, 2706466, Carrera 47 n. ${ }^{\circ} 37$ sur 18, Medellín, Colombia, correo electrónico: paola.botero@campusucc.edu.co

Cómo citar este artículo: Parra Quintero N, Botero Mariaca PM. Aparatos de avance mandibular: ¿mito o realidad? Rev. Nac. Odontol. 2013 diciembre; 9 (edición especial): $57-73$.

Resumen. La mayoría de los pacientes con maloclusión clase II esquelética presentan posición retruida de la mandíbula. El término aparato funcional se refiere a aparatos fijos o removibles diseñados para alterar la posición mandibular sagital y verticalmente, resultando en cambios esqueléticos, ortodónticos y ortopédicos. A pesar de su larga historia, los aparatos funcionales continúan siendo controversiales en su uso, efectividad y modo de acción. Esta revisión permitirá conocer las diferentes alternativas de tratamiento para la maloclusión clase II esquelética causada por retrusión mandibular, entre los que están Bionator, Twin Block, Herbst, Jasper Jumper, Forsus, MARA y Advansync, de los cuales se explicará su modo de acción, sus efectos a nivel dental y esquelético y el tiempo de tratamiento óptimo para conseguir estabilidad.

Palabras clave: avance mandibular, maloclusión de Angle clase II, mandíbula, retrognatismo.

\section{Mandibular AdVANCEMENT Apparatus: Myth or Reality?}

Abstract. Most patients with skeletal Class II malocclusion present a retruded position of the mandible. Functional appliances are fixed or removable appliances designed to alter the sagittal and vertical mandibular position, resulting in orthodontic and orthopedic skeletal changes. Despite their long history, functional appliances remain controversial in their use, effectiveness and mode of action. This review will reveal the different treatment options for skeletal Class II malocclusion caused by mandibular retrusion, such as: Bionator, Twin block, Herbst, Jasper jumper, Forsus, MARA and Advansync; it will also explain the mode of action, skeletal and dental effects and optimum treatment time for stability.

Keywords: mandibular advancement, class II Angle malocclusion, mandible, retrognatism. 


\section{Introducción}

La maloclusión clase II división 1 es un problema frecuente con una incidencia que varía entre el 5\% y el 29\% de los pacientes atendidos en la consulta de ortodoncia. Cerca de dos tercios de los pacientes con clase II división 1 es causada por la retrusión mandibular, la cual puede agravarse debido a que cerca de un tercio o la mitad de los pacientes con esta discrepancia presentan variaciones en el desarrollo vertical de los maxilares [1-4]. El concepto de ortopedia funcional mandibular o avance mandibular no es nuevo. Desde 1880, cuando Kingsley escribió acerca de "jumping the bite", se considera la posibilidad de adelantar la posición mandibular. Existe una evolución gradual del uso de la ortopedia funcional en la práctica ortodóncica, especialmente en el tipo de aparato empleado y el momento de aplicación del tratamiento [5]. A pesar de su larga historia, continúa existiendo mucha controversia acerca de su uso, método de acción y efectividad [6]. La literatura ortodóncica describe gran variedad de diseños de aparatos, incluyendo el Bionator, el Twin block, el Herbst, el Jasper jumper, el Forsus, el MARA y el Advansync, entre otros. Esta revisión se enfocará en el tratamiento de los problemas de maloclusión clase II que están relacionados principalmente con problemas esqueléticos, con énfasis en las mecánicas de reposicionamiento anterior mandibular, y especificando las diferencias encontradas en la corrección de dicha maloclusión en cuanto a los efectos esqueléticos y dentoalveolares. Este conocimiento nos permitirá llegar a una apropiada selección del aparato, el tiempo de tratamiento y la cooperación del paciente.

\section{Anatomía mandibular}

Etimológicamente el hueso mandibular proviene de la palabra mandere - que significa masticar-, es considerado como el hueso más grande y fuerte de los que conforman la cara y presenta una característica inusual por ser el único hueso móvil. En una visión lateral, el hueso mandibular se encuentra formado por una porción horizontal curva llamada cuerpo y dos porciones perpendiculares denominadas ramas; el sitio donde se une el cuerpo con las ramas constituye el ángulo mandibular (figura 1). Cada rama tiene un cóndilo en la parte posterior, que se articula con la fosa mandibular y con el tubérculo articular del hueso temporal para formar la articulación temporomandibular; también posee en la parte anterior las apófisis coronoides, importantes para la inserción del músculo temporal [7].
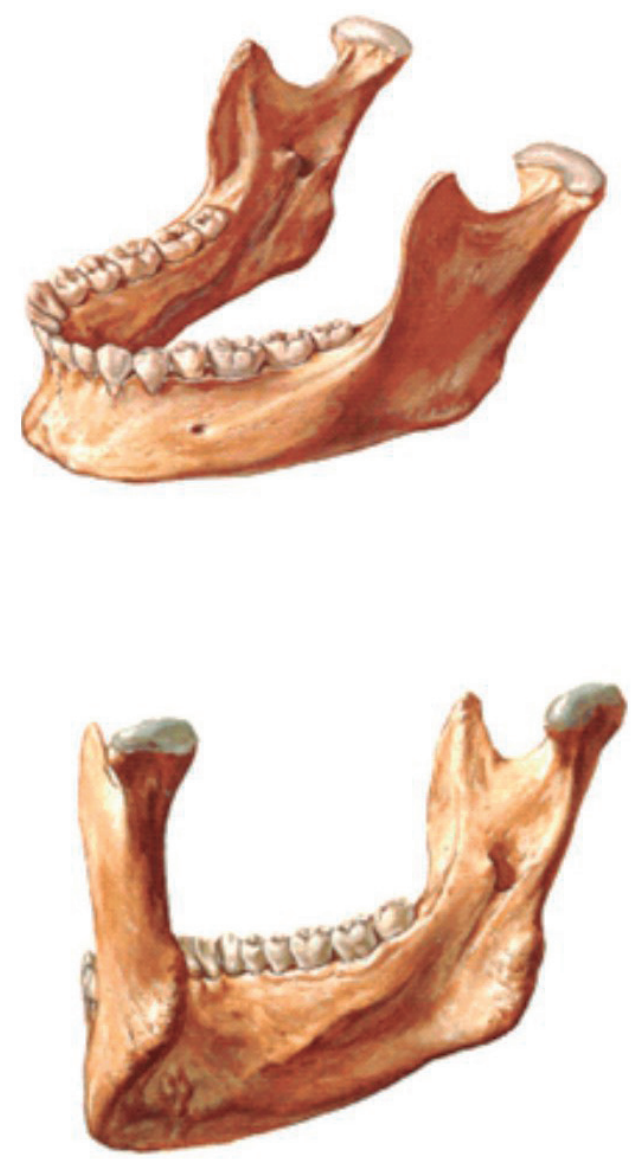

Figura 1. Mandíbula de un adulto: visión anterolateral izquierda y visión posterior izquierda

Fuente: Netter, 2007, 2009 [8]

La mandíbula se forma por osificación membranosa a partir del mesénquima del primer arco faríngeo, pero además desarrolla cartílagos secundarios en el cóndilo, en el proceso coronoídeo, en el ángulo mandibular y en la sutura intermaxilar (sínfisis). Estos cartílagos difieren en su origen, su estructura histológica y su respuesta a factores hormonales, metabólicos y mecánicos con respecto a los cartílagos primarios, presentes en los huesos largos [9].

\section{Patrón de crecimiento mandibular}

El crecimiento mandibular es un proceso complejo que involucra diferentes procesos como desplazamiento antero-inferior, recolocación de la rama ascendente, crecimiento del cuerpo mandibular en longitud y varios tipos de rotaciones óseas. 
La remodelación condilar y de la rama son relativos al desplazamiento mandibular, el cual puede ser primario o secundario; el primario es generado por un crecimiento intrínseco del hueso, mientras que el secundario se genera por influencia del crecimiento de las regiones laterales de la base craneal (principalmente la sincondrosis esfenooccipital) [10], lo que desplaza la fosa glenoidea hacia la parte anterior afectando indirectamente el grado de protrusión mandibular -en $3 \mathrm{~mm}$ aproximadamente- con un incremento de la distancia entre Nasion y la articulación temporomandibular - en un promedio de 7,5 $\mathrm{mm}-\mathrm{y}$ por tanto un cambio en el perfil facial.

Al desplazarse la mandíbula hacia abajo y adelante se produce una rotación entre 0,5 y 1,0 grados por año [11], la cual depende del patrón de crecimiento condilar y las cargas funcionales. Las rotaciones adelante y arriba, por ejemplo, muestran grandes cantidades de crecimiento condilar en la parte anterior, lo que incrementa la altura vertical [11] con un nivel alto de neoformación-reabsorción y un potencial de crecimiento rápido y extenso. En los rotadores hacia abajo y atrás se incrementa el crecimiento condilar en la parte posterior, lo cual incrementa la longitud sagital con un nivel bajo de neoformación-reabsorción y un potencial de crecimiento disminuido. Estos cambios en las regiones del cóndilo mandibular explican por qué los rotadores arriba y adelante se caracterizan por un ángulo mandibular pequeño que disminuye durante el crecimiento [11-13].

El crecimiento sagital de la mandíbula ha sido estudiado con métodos histológicos y estudios clínicos con implantes entre los 10 y 15 años, en los que se ha establecido que el crecimiento mandibular en longitud ocurre principalmente en el cóndilo. Dicha estructura crece superior y levemente posterior o incluso anteriormente, dependiendo de la orientación y el patrón de crecimiento individual [14]. Los patrones de remodelación de la rama y el cuerpo involucran aposición ósea a lo largo del borde posterior de la rama y reabsorción en el borde anterior, mientras el proceso gonial muestra una gran cantidad de remodelación posterior y superior [12, 15]. En el borde inferior ocurre reabsorción posterior y aposición anterior $[10,15]$. Por otro lado, la sínfisis se amplía por aposición posterior, superior e inferior [11]. Gracias a estos patrones de remodelación el mentón se mueve hacia abajo y atrás, mientras que el punto B se desplaza en sentido superior y posterior $[10,11,15]$ (figura 2).

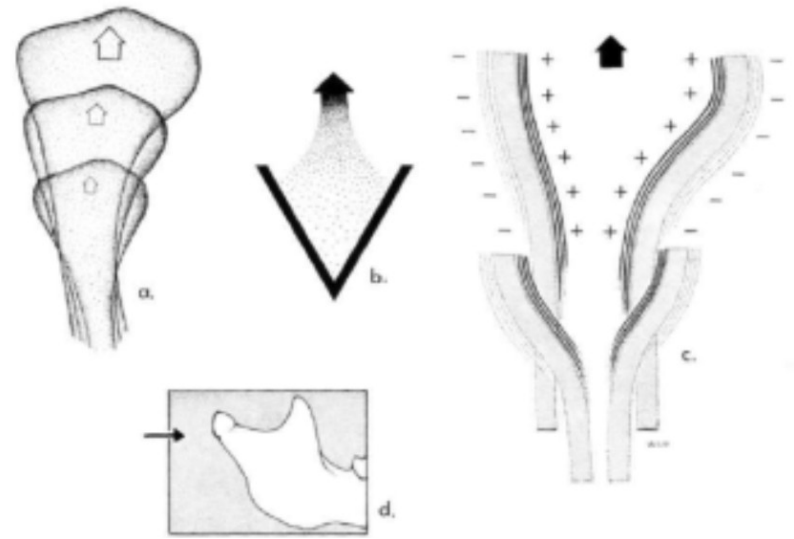

Figura 2. El diámetro del cuello del cóndilo es estrecho (d) y se reduce progresivamente desde la dimensión más amplia del movimiento posterior del cóndilo (a), el crecimiento hacia el interior de las corticales vestibular y lingual (c) se realiza por un proceso de combinación de resorción perióstica(-) y deposición endósti$\mathrm{ca}(+)$. Este es un ejemplo del principio $\mathrm{V}(\mathrm{b})$

Fuente: Enlow, Harris, 1964 [16]

El crecimiento transversal se ve limitado por el cierre temprano de la sínfisis; sin embargo, varios estudios han mostrado un incremento en el ancho mandibular desde $0,28 \mathrm{~mm}$ hasta $0,4 \mathrm{~mm}$ por año entre los 4 y los 20 años de edad, lo que equivale al 65\% de la ampliación del maxilar (figura 3) [12]. La amplitud bigonial presenta un incremento de $13,7 \mathrm{~mm}$ en hombres y $12,9 \mathrm{~mm}$ en mujeres entre los 7 y 15 años [13].

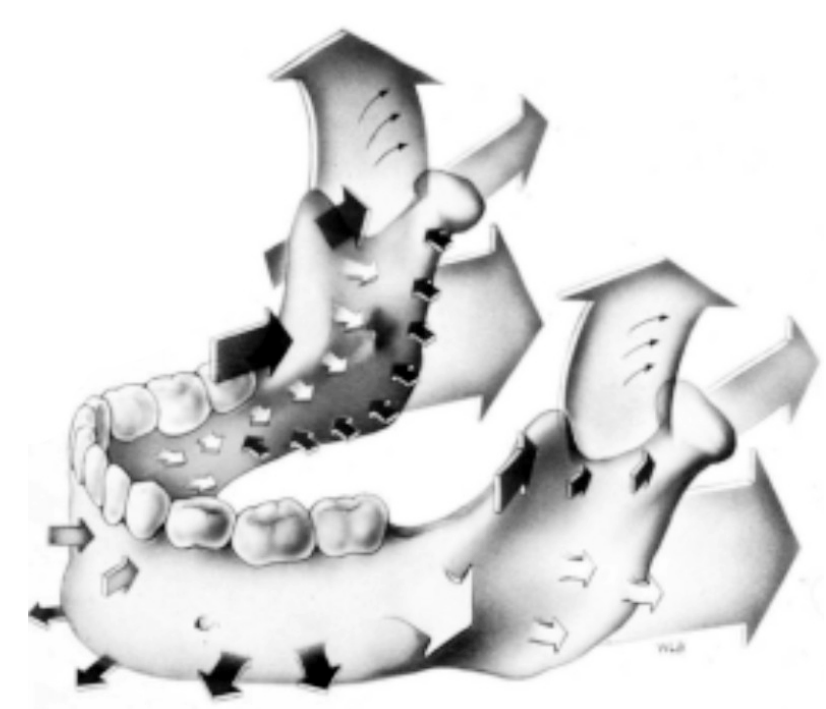

Figura 3. La mandíbula como un conjunto, el cual representa una multitud de sitios activos de crecimiento que comprenden todas las regiones del periostio y endostio a través del hueso

Fuente: Enlow, Harris, 1964 [16] 
Durante el crecimiento mandibular se presentan varios picos de crecimiento que ocurren al mismo tiempo que los del crecimiento general; el primero ocurre desde el nacimiento hasta los tres años de edad, el segundo es observado entre los 6 y 7 años en niñas y de 7 a 9 años en niños. El tercer pico es denominado circumpuberal y, como su nombre lo indica, ocurre cercano a la pubertad y no concuerda con una edad cronológica específica, aunque algunos autores la asocian con un promedio de edad de 11 a 12 años en mujeres y de 14 a 15 años en hombres [17]. Este último pico de crecimiento es el periodo de máxima aceleración de desarrollo óseo, que coincide con la aparición de las características sexuales secundarias [18]. La mandíbula continúa alargándose 2 años después de que el maxilar desacelera su crecimiento, y en promedio crece en total de 24 a $33,5 \mathrm{~mm}$.

En el potencial de crecimiento de la mandíbula existen diferencias entre los sexos: los varones tienen un mayor potencial de crecimiento que las mujeres entre los 10 y 14 años debido a un pico de crecimiento adolescente más intenso, y aproximadamente dos años más de crecimiento debido a las diferencias de maduración. El patrón de crecimiento del hombre muestra un perfil facial recto a medida que crece, mientras que la mujer muestra un menor crecimiento incremental y duración del crecimiento de la mandíbula, por lo que su perfil se mantiene más convexo en comparación con los hombres [19].

\section{El papel del cóndilo en el desarrollo}

El cartílago condilar es un tipo de cartílago secundario que aparece una vez iniciada la osificación intramembranosa y no por diferenciación desde el cartílago primario como está establecido en el cráneo y en los huesos largos. Por ser secundario, posee la capacidad de adaptación a la compresión regional como respuesta específica ante estímulos locales (tolerancia a la presión), debido a que no contiene programación genética que determina y gobierna directamente el curso de crecimiento. Su contribución es proporcionar el crecimiento regional adaptativo al mantener la relación anatómica adecuada entre el hueso temporal y el cóndilo mientras la mandíbula está siendo llevada simultáneamente hacia abajo y adelante. El cóndilo no establece el rango o la cantidad de crecimiento mandibular, pero tiene la capacidad especial de crecimiento y remodelación multidireccional en respuesta a los movimientos y las rotaciones variadas generadas por el desplazamiento mandibular (tabla 1).

Tabla 1. Diferencias en la formación de los cartílagos

\begin{tabular}{|l|l|l|}
\hline Tipo de cartílago & \multicolumn{1}{|c|}{ Ubicación en el cuerpo } & Patrón de formación ósea \\
\hline $\begin{array}{l}\text { Cartílagos } \\
\text { primarios }\end{array}$ & $\begin{array}{l}\text { Epífisis, sincondrosis } \\
\text { esfenooccipital y tabique nasal. }\end{array}$ & $\begin{array}{l}\text { La matriz cartilaginosa aísla los condroblastos en división de aquellos } \\
\text { factores locales que pueden limitar o estimular la velocidad de crecimiento } \\
\text { condral. }\end{array}$ \\
\hline $\begin{array}{l}\text { Cartílagos } \\
\text { secundarios }\end{array}$ & $\begin{array}{l}\text { Cóndilo, coronoides y } \\
\text { cartílagos de algunas suturas } \\
\text { craneofaciales. }\end{array}$ & $\begin{array}{l}\text { Las células en proceso de división no están rodeadas por la matriz } \\
\text { cartilaginosa, y por consiguiente no están aisladas de la influencia de factores } \\
\text { locales (los factores extrínsecos locales pueden modificar la velocidad de su } \\
\text { crecimiento. }\end{array}$ \\
\hline
\end{tabular}

Fuente: Graber y Petrovic, 2009 [5]

El ritmo y la dirección del crecimiento condilar están presumiblemente sujetos a la influencia de agentes extracondilares, incluyendo fuerzas biomecánicas intrínsecas y extrínsecas. Se cree que cantidades crecientes de presión sobre el cartílago sirven para inhibir el ritmo de división y el crecimiento celular, mientras que cantidades disminuidas estimulan y aceleran el crecimiento [15].

\section{Patrón de crecimiento de pacientes clases II esquelética}

Los individuos clase II presentan una discrepancia en el crecimiento sagital maxilo-mandibular (el crecimiento maxilar puede estar aumentado, el mandibular 
disminuido o una combinación de ambos [20], que puede agravarse por alteraciones en el crecimiento vertical. Por otro lado, los patrones funcionales de cada individuo (respiración, deglución y masticación, entre otras) pueden alterar la forma y el patrón de crecimiento [21]. Es importante saber que los patrones esqueléticos se establecen desde los 6 años de edad, y pueden empeorar con el crecimiento al incrementarse la deficiencia mandibular o el prognatismo maxilar [22, 23].

Anatómicamente los pacientes clase II pueden tener diferencias con los pacientes clase I. Los pacientes clase II tienen la fosa glenoidea ubicada más posterior y con una forma más amplia y superficial $[14,21]$. Por otro lado, el cóndilo se encuentra más elongado e inclinado hacia adelante [19], y presentan una base craneal anterior larga y con un ángulo agudo. Además, la cantidad de crecimiento anual en estos pacientes se encuentra disminuida (de 0 a 1,2 mm anuales). Cefalométricamente se ha encontrado que el maxilar superior se encuentra más protruido en los pacientes clase II (sNA aumentado) y la posición mandibular retruida (sNB disminuido) entre los 10 y 14 años [22]. La rotación mandibular en este tipo de pacientes puede ser hacia abajo y atrás (patrón de crecimiento vertical) o arriba y adelante (patrón de crecimiento horizontal) (tabla 2). Ambos patrones presentan una respuesta diferente ante las fuerzas extrínsecas: los rotadores verticales presentan una respuesta desfavorable ("malos crecedores"), mientras que los rotadores horizontales experimentan una respuesta favorable en su crecimiento ("buenos crecedores"), ya que responden positivamente ante fuerzas aplicadas $[24,25]$.

Tabla 2. Características esqueléticas de pacientes con crecimiento horizontal versus pacientes con crecimiento vertical

\begin{tabular}{|l|l|}
\hline \multicolumn{1}{|c|}{ Pacientes con crecimiento horizontal } & \multicolumn{1}{|c|}{ Pacientes con crecimiento vertical } \\
\hline Rotación anterior mandibular. & Rotación posterior mandibular. \\
\hline El cóndilo crece en sentido más anterior. & Cóndilo crece en sentido posterior. \\
\hline Rama mandibular ancha. & Rama mandibular angosta. \\
\hline Ángulo goniaco cerrado (Co-Go-Me) menor a 125,5 grados & Ángulo goniaco abierto (Co-Go-Me) mayor a 125,5 grados. \\
\hline Sínfisis y bases alveolares bajas y amplias en sentido transverso. & Sínfisis y bases alveolares altas y angostas en sentido transverso. \\
\hline
\end{tabular}

Fuente: Bishara y Guardo, 2003 [26]

La forma como se relacionan los arcos dentales entre sí desencadena en determinados casos una señal que modifica la actividad del músculo pterigoideo lateral y de otros músculos de la masticación, permitiendo que el maxilar superior se ajuste a la posición oclusal óptima. Es probable que este cambio en la actividad del músculo pterigoideo lateral influya en la velocidad de crecimiento del cartílago condíleo. En alguna medida, los aparatos funcionales pueden desencadenar respuestas condíleas parecidas.

\section{Efectos de la estimulación de crecimiento mandibular a nivel condilar, cavidad glenoidea y muscular}

En las respuestas del cóndilo, la cavidad glenoidea y la muscular existen diferencias ante estímulos externos entre las distintas regiones anatómicas, donde algunas son más susceptibles que otras a la modificación de los factores epigenéticos locales y el medio ambiente 
[5]. Experimentos in vitro realizados desde 1968 han demostrado que algunos músculos masticatorios estimulados por aparatos ortopédicos pueden modificar la velocidad y magnitud del crecimiento del cartílago condíleo [5]. Por otro lado, estudios clínicos también han demostrado que el crecimiento del cóndilo es sensible a los cambios en la posición mandibular adaptándose a la función protrusiva mediante un crecimiento posterior, mientras que el incremento en la región gonial posterior representa una compensación ocasionada por el posicionamiento anterior mandibular [27] (figura 4).

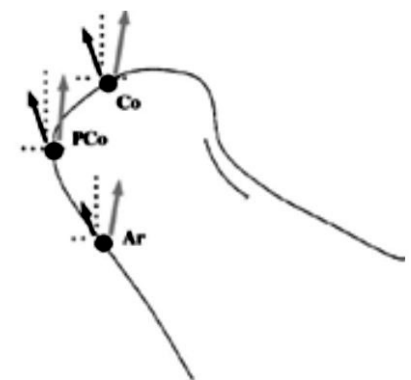

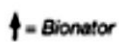

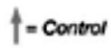

b.

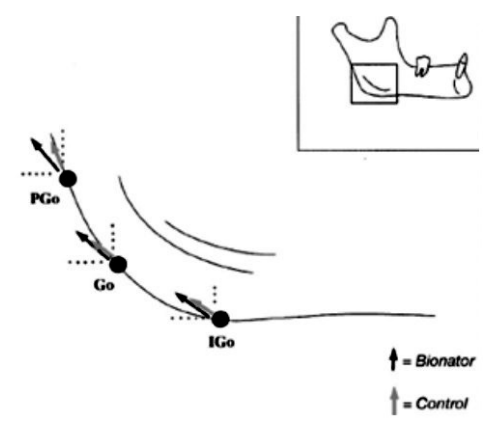

Figura 4. (a) remodelación mandibular y (b) crecimeinto condilar después de 1 año para los niños con maloclusion clase II tratados y no tratados con bionator $(+=0,5 \mathrm{~mm})$

Fuente: Araujo, Buschang y Melo, 2004 [27]

Otros estudios sugieren efectos a nivel de la cavidad glenoidea con formación de nuevo hueso en la zona posterior [28]. El posicionamiento anterior mandibular guía a un incremento significativo en la cantidad de expresión del factor de crecimiento vascular endotelial (VEGF) en la fosa glenoidea cuando se compara con crecimiento natural en un grupo control no tratado, lo que conlleva a un aumento significativo en la formación de hueso nuevo [29] (figura 5). a.

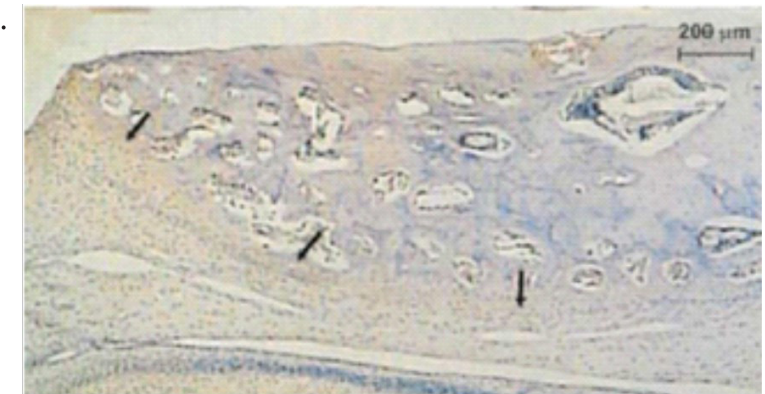

b.

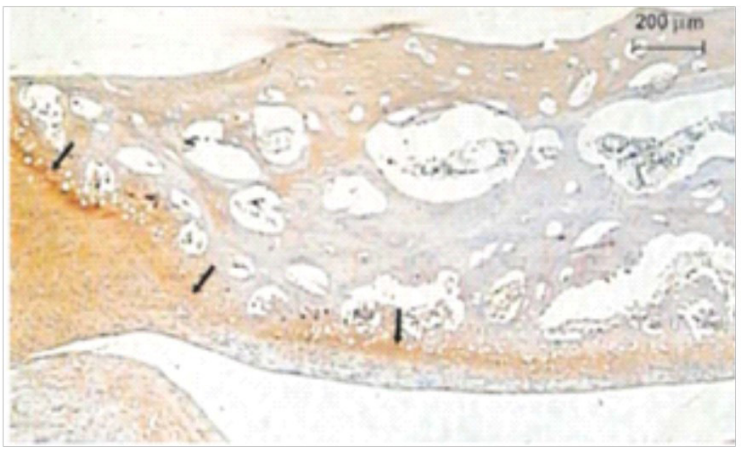

Figura 5. Expresión positiva de vEGF en la fosa glenoidea en ratas de 49 días. a) Durante el crecimiento natural, y b) después de 14 días de avance mandibular

Fuente: Rabie, Shum y Chayanupatkul, 2002 [29]

El efecto de los aparatos funcionales a nivel muscular también ha sido ampliamente estudiado; por ejemplo, en 1975 McNamara encontró que estos pueden modificar la velocidad y cantidad de crecimiento condilar mediante la estimulación de la actividad del músculo pterigoideo lateral, y Voudouris reportó en el 2000 la hipótesis de la relatividad del crecimiento, en la cual explica que el desplazamiento mandibular esquelético, dental o neuromuscular, la tensión de tejidos viscoelásticos de la ATM y fuerzas a través del fibrocartílago entre fosa-cóndilo generan cambios condilares [30, 31]. Sin embargo, un estudio realizado en ratas sometidas a pterigoidectomía unilateral mostró que la presencia o ausencia del pterigoideo lateral tuvo poco efecto sobre el crecimiento condilar [32,33]. Al obligar al paciente a asumir una posición adelantada de la mandíbula se produce tensión y estiramiento de los músculos elevadores con una subsecuente adaptación ósea y muscular en sentido sagital [33]. Los estudios que evalúan la actividad electromiográgica (EMG) durante el tratamiento con aparatos funcionales, muestran disminución de esta después del primer mes, lo que persiste por aproximadamente 6 semanas. Entre el 
tercer y sexto mes es cuando ocurren las mayores adaptaciones y se logran los mayores contactos oclusales y por tanto se incrementa la actividad EMG. Con estos hallazgos se demuestra que los pacientes no presentan dificultad en adaptarse a la nueva posición mandibular después de 6 meses de tratamiento activo. Sin embargo, es importante aclarar que no todos los estudios presentan uniformidad en cuanto al diseño vertical de los aparatos empleados y por tanto se pueden encontrar aumentos y disminuciones de las actividades electromiográficas [34].

\section{Aparatos funcionales}

Para tratar las maloclusiones clase II frecuentemente se recurre a la aplicación de una fuerza extraoral sobre el maxilar superior. No obstante, son numerosas las investigaciones que demuestran que una gran parte del problema reside en el maxilar inferior - que está insuficientemente desarrollado- o posiblemente en una posición retruida, o ambas cosas [5]; por tanto, surge el deseo de modificar su posición.

El término "aparato funcional" se refiere a una variedad de aparatos removibles diseñados para alterar la disposición de varios grupos de músculos - que influencian la función y posición de la mandíbula para trasmitir fuerzas a la dentición y el hueso basal- mediante un mecanismo de acción indirecta al promover una reacción muscular, la cual ejerce la fuerza necesaria para obtener cambios dentales o esqueléticos. Típicamente estas fuerzas musculares son generadas por la alteración de la posición mandibular sagital y verticalmente, resultando en cambios ortodónticos y ortopédi$\cos [6,35]$. La terapia con aparatos funcionales permite obtener un promedio de $6 \mathrm{~mm}$ de corrección para la maloclusión clase II con una combinación de efectos ortopédicos (30\% a 40\%) - tales como incremento de la longitud mandibular (co-pg), incremento en la amplitud de la rama (co-go), apertura del ángulo goniaco (ar-goi-me), rotación posterior de la línea condilar en relación con la línea mandibular (cl-ml) y ausencia de desplazamiento adelante de la cabeza del cóndilo [36] - y dentoalveolares (60\% a 70\%), como extrusión de los molares maxilares y mandibulares, vestibularización de incisivos inferiores y lingualización de los superiores [6]. Los mayores efectos ortopédicos se esperan cuando el tratamiento es llevado a cabo en el pico de crecimiento mandibular, cuando se comparan con los resultados de tratamiento realizados antes y después del pico de crecimiento [37]. Con respecto a los efectos en la ATM, se encuentra que la almohadilla retrodiscal recibe un estímulo mucho más intenso, al estar el maxilar inferior en una posición más adelantada. El aumento y la reiteración de la actividad de la almohadilla retrodiscal adelantan el comienzo de la hipertrofia de los condroblastoscondileos y, por consiguiente, incrementan la velocidad de crecimiento del cartílago [5]. La respuesta mandibular ante el estímulo depende del patrón de crecimiento vertical del paciente. En pacientes considerados como "buenos crecedores", se encuentra un aumento semestral de Co-Gn mayor a $5,3 \mathrm{~mm}$ (comparado con un paciente clase II sin tratamiento, cuyo aumento de la longitud mandibular es de $3,3 \mathrm{~mm}$ ), mientras que en los "malos crecedores" este aumento es menor $[37,38]$.

La ortopedia funcional busca modificar el sistema de fuerzas del sistema masticatorio para producir cambios remodelativos óseos y dentoalveolares. La alteración de las fuerzas durante la posición postural parece ser la más importante para la inducción de cambios remodelativos estructurales $[39,40]$. Existen muchos diseños de aparatos que dependen de la teoría de acción que posea su diseñador acerca de estos. Los aparatos funcionales más empleados son el bionator y el Twin Block, los cuales describiremos en los siguientes apartados.

\section{Bionator}

Es un aparato bimaxilar desarrollado por el alemán William Balters en 1968, basado en el activador de Andreasen. Para el diseño se le otorgó más importancia al papel que desempeña la lengua en la cavidad bucal y su reeducación, considerando que tanto esta como los músculos periorales son los responsables de las formas de las arcadas dentales [5, 41]. Está encaminado a modular la actividad muscular, favoreciendo el desarrollo normal al suprimir factores ambientales anormales y estimulando la actividad refleja miotática con la contracción isotónica de los músculos, lo que genera cambios dentoalveolares sagitales y verticales (figura 6) [5]. 

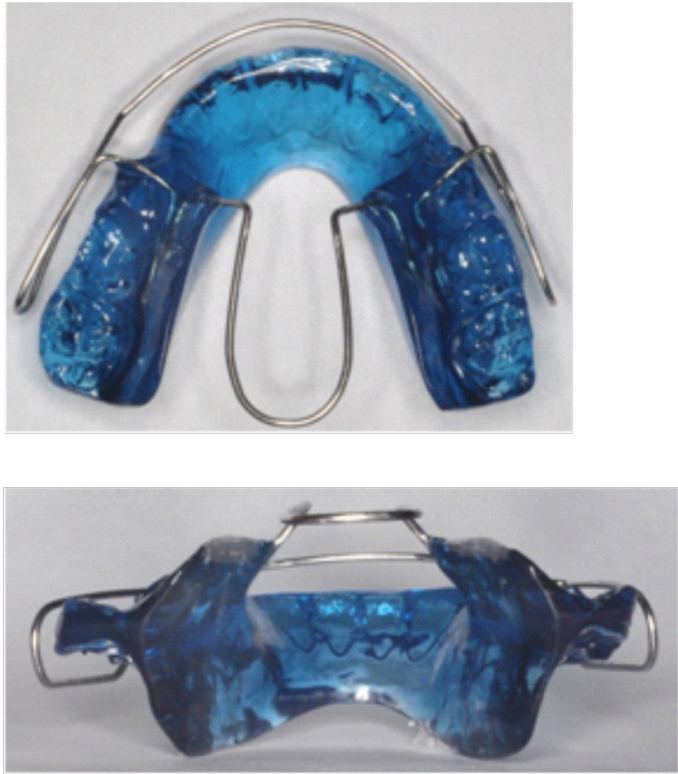

Figura 6. Vista oclusal y frontal del bionator Fuente: cortesía de la doctora Andrea Ocampo

Los cambios generados con este tipo de aparato aplicado durante el pico de crecimiento son en su mayoría a nivel dentoalveolar con retroinclinación de incisivos maxilares, proinclinación inferiores (con una corrección en el overjet de 1,6 a 4,4 mm) y en la relación molar. A nivel esquelético se encontró un aumento de $4,3 \mathrm{~mm}$ en la longitud total de la mandíbula y en la altura de la rama, junto con una apertura significativa del ángulo mandibular e incremento en altura facial anteroinferior [38]. La terapia con bionator desplaza la mandíbula anteriormente, limita la cantidad de rotación anterior y cambia la dirección del crecimiento condilar, pero no incrementa la cantidad de crecimiento (produce aposición ósea en la región condilar y gonial) [27, 41]; mientras que a nivel maxilar no se encuentra ninguna modificación significativa [41, 42]. Varios autores sugieren crecimiento mandibular con el aparato funcional. El aparto se debe usar día y noche con controles de 3 a 5 semanas, con un promedio de duración del tratamiento de 12 meses [5].

\section{Twin Block}

Aparato funcional empleado para la corrección de las desarmonías esqueléticas y oclusales de pacientes clase II caracterizados por la retrusión mandibular. Fue desarrollado originalmente por el escocés William J. Clark y consiste en unos platos acrílicos maxilares y mandibulares con planos de mordida que obligan a la mandíbula a ir hacia adelante en el cierre produciendo adaptaciones tanto esqueléticas como dentoalveolares [43] (figura 7). El principal objetivo es inducir un alargamiento complementario de la mandíbula por el estímulo de crecimiento en el cartílago condilar aplicado idealmente durante el pico de crecimiento puberal, aunque los tratamientos más tempranos tienen efectos de menor magnitud.

El tratamiento iniciado durante el pico de crecimiento produce efectos más favorables que incluyen: mayor contribución del esqueleto a la corrección molar (avance del mentón y pogonion de 2,5 mm), mayor incremento en longitud total mandibular $(4,75 \mathrm{~mm} /$ año versus $1,88 \mathrm{~mm} /$ año en pacientes antes del pico) y en la altura de la rama, dirección más posterior del crecimiento del cóndilo [36], reducción del overjet (6 $\mathrm{mm}$ por año durante el pico y $4,5 \mathrm{~mm}$ por año antes del pico) e inhibición leve del crecimiento maxilar sagital [43]. A nivel condilar se reporta una rotación hacia atrás del mismo y un crecimiento adicional en una dirección posterosuperior, con incremento en la aposición de hueso en los aspectos posteriores de la cabeza del cóndilo y de la rama.

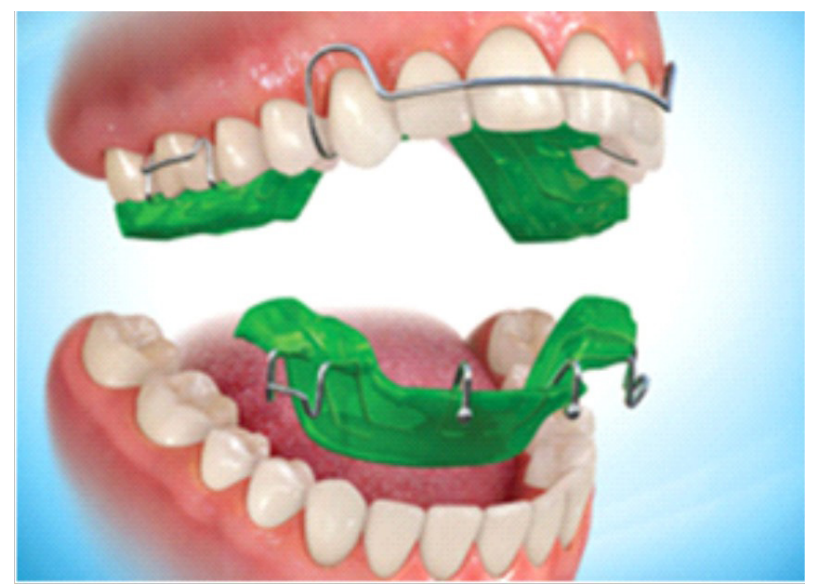

Figura 7. Platos acrílicos maxilares y mandibulares con planos de mordida

Fuente: Catalogo Dentaurum [44]

Con respecto a los cambios dentoalveolares, se reporta que la mayor contribución en la corrección del overjet se representa por la protrusión de incisivos inferiores $(0,7 \mathrm{~mm})$ y una proinclinación de estos $(2,8$ grados en promedio, con un incremento de $1,4 \mathrm{~mm}$ por año en el grupo tratado tempranamente y de 2,2 $\mathrm{mm}$ por año en el grupo tratado tardíamente) y una retroinclinación de los superiores (-4,3 grados) gracias al contacto permanente del labios superior sobre ellos. El movimiento distal de los molares superiores $(1,5$ $\mathrm{mm}$ ) y el movimiento mesial de los molares inferiores 
contribuyó a la corrección de la relación molar $(4,8$ $\mathrm{mm}$ ) en ambos grupos tratados $[40,43]$.

El control de la dimensión vertical es uno de los beneficios del twin block, debido a que los bloques de mordida inhiben la erupción de los molares en pacientes que tienen una altura facial anterior inferior aumentada. Sin embargo, en otros casos reportan que los molares inferiores erupcionan en promedio 4 veces más ( $2,3 \mathrm{~mm}$ en total) con incrementos significativos en la altura facial anterior $(3,8 \mathrm{~mm})$ y la altura facial posterior $(2,9 \mathrm{~mm})$ [39].

Para lograr los efectos antes descritos, el uso del aparato debe ser de 24 horas durante 9 a 12 meses, con adecuada estabilidad de los resultados al evaluar los pacientes tres años después $[36,39,40]$.

\section{Aparatos ortopédicos}

La palabra ortopedia viene de las raíces griegas ortos, que significa recto, y paideia, que significa educación. Teóricamente todo tratamiento que busque el mejoramiento craneofacial de un individuo durante su desarrollo pertenece a la categoría de ortopédico. Sin embargo, la colocación del adjetivo "funcional" limita la aplicación de este término a las terapias que buscan regular o modificar la función para alterar el tamaño y la forma facial. Aunque hay un gran número de aparatos que proclaman tener efectos sobre la función del sistema craneofacial - al modificar la posición mandibular generando una corrección estructural del retrognatismo- no producen un cambio funcional como tal sino una posición forzada anterior de la mandíbula mediante la aplicación de fuerzas ortopédicas [44]. Los aparatos ortopédicos más utilizados son Herbst, Jasper Jumper, Forsus, MARA y Advansync, los cuales describiremos en los siguientes apartados.

\section{Herbst}

Fue el primer aparato ortopédico diseñado por Emil Herbst en 1905; sin embargo, fue modificado y más usado por Hans Pancherz desde finales de 1970, quien pretendía lograr un crecimiento condilar efectivo. Es un aparato fijo y rígido que requiere de un complejo proceso de laboratorio [31] (figura 8). Consta de un mecanismo telescópico que permite el reposicionamiento anterior de la mandíbula cuando el paciente está en oclusión, liberando aproximadamente una fuerza de 200-250 gr durante el cierre $[45,46]$.

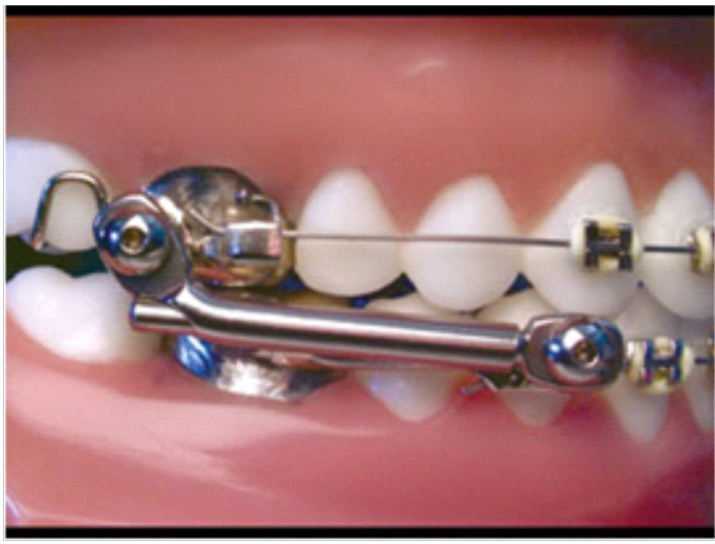

Figura 8. Vista lateral del aparato de Herbst con bandas en los primeros molares maxilares y mandibulares unidos por un mecanismo telescópico

Fuente: Pancherz y Ruf, 2008 [47]

Los estudios clínicos muestran efectos esqueléticos y dentoalveolares. Cerca del 50\% de los efectos del tratamiento se dan por movimiento dental, principalmente por el movimiento adelante y arriba de la dentición posterior. Esqueléticamente hay un incremento en el crecimiento mandibular $(2,0 \mathrm{~mm}$ a 2,5 $\mathrm{mm}$ más de los valores normales y un avance del mentón de $2,5 \mathrm{~mm}$ ), pero sólo a corto plazo [45-48], con una eficacia del $90 \%$ en la corrección de la clase II por disminución en el ANB, en el overjet y en el overbite, además de una rotación posterior mandibular. La cantidad de aumento en la longitud mandibular depende del diseño del Herbst; en los casos de aparato con bandas se reporta un incremento de 2,0 a 2,2 mm a los 6 meses, y para el aparato con férula acrílica se reporta un incremento de 2,7 a 3,5 $\mathrm{mm}$ al año de tratamiento [49]. Este incremento es menor en casos de pacientes iniciados antes del pico de crecimiento [49].

Los incrementos en la protrusión mandibular durante el tratamiento se les atribuyen a tres procesos adaptativos en la ATM: (1) incremento en el crecimiento condilar debido a su remodelación, (2) desplazamiento anterior de la fosa glenoidea gracias a un proceso de aposición y reabsorción y (3) posicionamiento anterior del cóndilo. El cóndilo crece en una dirección postero-superior y la fosa glenoidea presenta un desplazamiento anterior resultando en un desplazamiento del pogonion hacia adelante $[48,50,51]$. Verticalmente se genera un incremento de $1,8 \mathrm{~mm}$ en la altura facial inferior sólo en la zona anterior [52]. En el maxilar no se encuentran cambios clínicamente significativos, sólo una pequeña restricción de su crecimiento de $0,9^{\circ}$ en el ángulo sNA y de 1,2 $\mathrm{mm}$ en la restricción del desplazamiento sagital del punto A [49]. 
Dentoalveolarmente ocurre un movimiento distal de los molares maxilares de $-1,7 \mathrm{~mm}$ por año [36], con una retracción significativa de los incisivos maxilares de $1,4 \mathrm{~mm}$ [49] y una intrusión y protrusión de los incisivos inferiores de $10,8^{\circ}$ (el borde incisal se mueve anteriormente $3,2 \mathrm{~mm}$ ). Sin embargo, después de 6 meses postratamiento la inclinación del incisivo inferior recidiva en promedio $7,9^{\circ}$ y el borde incisal se mueve posteriormente $2,5 \mathrm{~mm}[49,53,54]$. El Herbst no es un aparato para usar en pacientes con dentición mixta. Los dientes posteriores deciduos tienden a ser planos y por esto no se cuenta con el mismo tipo de interdigitación oclusal como ocurre en la dentición permanente; por tanto, puede haber tendencia a una recidiva significativa hacia la maloclusión original. Ruf y Pancherz encontraron que el periodo ideal para el tratamiento con Herbst es en la dentición permanente o justo después del pico de crecimiento puberal. El tiempo de tratamiento es en promedio es de 8 meses, seguido de una terapia de ortodoncia interceptiva correctiva, para completar un tiempo total de tratamiento de 18 meses $[17,51]$.

\section{Jasper jumper}

Aparato ortopédico diseñado por James Jasper en 1987 [55] con un mecanismo y diseño nuevos para mayor flexibilidad. Se aplican fuerzas posteriores en la dentición maxilar y fuerzas anteriores recíprocas en la dentición mandibular [56]. Es ideal en maloclusiones clase II con mordida profunda con exodoncias y sin exodoncias (figura 9).

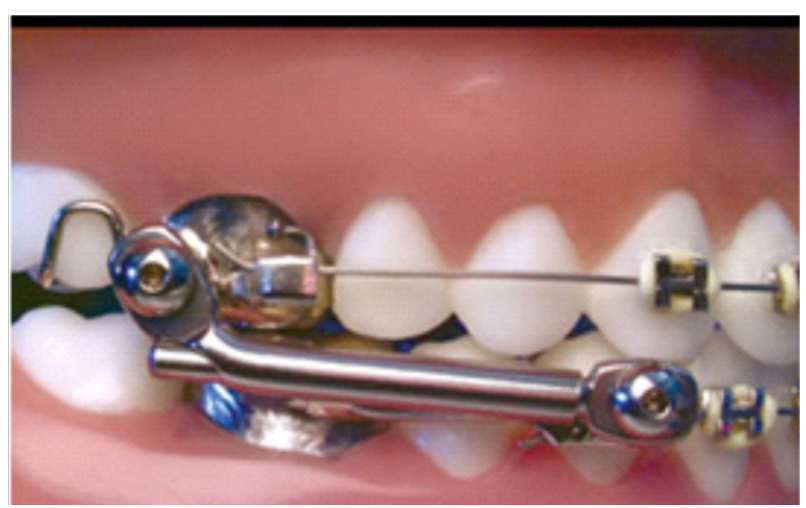

Figura 9. Jasper Jumper en posición

Fuente: Catalogo American Orthodontics [58]

Es un aparato que mantiene la mandíbula en una posición protruida aplicando fuerzas continuas y leves. Se activa cuando se abre la boca, pero esta activación se libera cuando se cierra total o parcialmente generando una fuerza de aproximadamente 60-250 gr. Cuando se activa $4 \mathrm{~mm}$, puede generar fuerzas mayores alrededor de 360 grs. Se recomienda su uso en la etapa de dentición permanente temprana [57].

Su mecanismo de acción puede ser como el de una tracción extraoral maxilar o un activador o una combinación de ambos dependiendo de cómo sea activado [59]. A pesar de su modo de acción distinto, el Jasper jumper tiene efectos similares al Herbst, incluyendo la restricción del crecimiento anterior del maxilar, la retracción dentoalveolar de la dentición maxilar, el movimiento distal de los molares maxilares, la protracción dentoalveolar mandibular, la protrusión mandibular y la mejoría en las relaciones maxilomandibulares, con la ventaja de permitir movimientos de lateralidad de la mandíbula, buena cooperación y fácil higiene oral; sin embargo, sigue siendo un aparato débil y los efectos ortopédicos de aumento en el crecimiento mandibular no se han comprobado [55].

Weiland y Bantleon reportaron que la corrección de la maloclusión clase II fue lograda por cambios esqueléticos (40\%) y dentales (60\%). Los efectos esqueléticos en el maxilar se reducen a generar una resistencia al crecimiento hacia adelante del maxilar [55, 59, 60]; a nivel mandibular se ve un incremento no muy significativo en la longitud mandibular con una mejoría en las relaciones maxilomandibulares [55]. Dentro de los efectos dentales se encuentran una disminución del overjet y el overbite gracias a la retroinclinación de incisivos superiores $[55,59]$ y distalización de los molares con inclinación e intrusión de estos. Los incisivos inferiores se proinclinan e intruyen como efectos indeseables [55] que podrían ser contrarrestados con el uso de brackets con torque coronal negativo. Los incisivos también se intruyen, lo que genera una rotación abajo y atrás del plano oclusal [59]. Algunos artículos reportan un movimiento mesial y de extrusión de los molares mandibulares durante el uso del aparato, lo que ayuda a la corrección sagital de la maloclusión en 4,52 $\mathrm{mm}$. Facialmente el labio superior se mueve hacia atrás y el labio inferior muestra protrusión $[55,60]$.

La preparación apropiada del anclaje es crítica para lograr resultados exitosos. Se debe colocar un arco de acero de 0,017 " X 0,025", o 0,018" X 0,025" (especialmente en el arco inferior) antes de adaptar el Jasper Jumper, para evitar el movimiento no deseado mesial del incisivo inferior y distal del molar superior. La fase 
de nivelación y preparación de anclaje se demora alrededor de 6 meses y de 6 a 9 meses de uso del aparato, con 3 a 4 meses de retención adicional para lograr adecuada estabilidad [57].

\section{Forsus (Forsus Fatigue Resistant Device)}

Aparato ortopédico desarrollado por Bill Vogt en el 2001, que consiste en un sistema telescópico semirrígido incorporado a un resorte de níquel titanio (que produce aproximadamente 150 a 200 gr de la fuerza cuando está totalmente comprimido) y que puede ser usado en conjunto con la aparatología fija $[57,61,62]$ (figura 10).

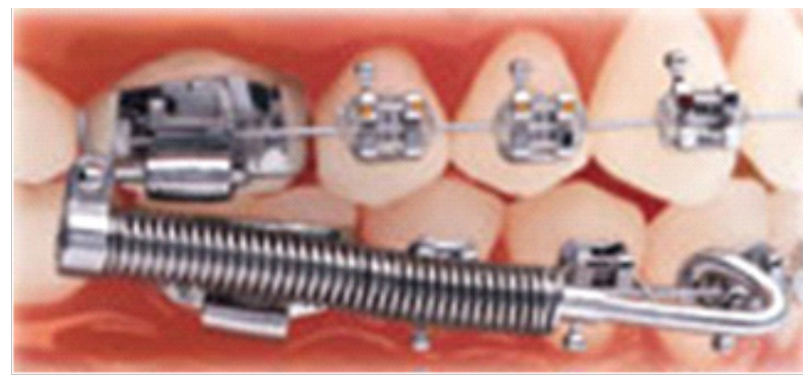

Figura 10. Vista lateral del forsus

Fuente: 3M Unitek Colombia, 2012 [65]

El forsus está adherido al primer molar maxilar y al arco mandibular - distal al canino o al bracket del primer premolar [61] - compuesto por barras espirales de níquel titanio unidos con un plástico trasparente para evitar la interferencia de las mejillas. Es un aparato más flexible y elástico que el Herbst, en el que la mandíbula puede realizar fácilmente movimientos laterales; los pacientes pueden cerrar en relación céntrica, morder repetidamente y deglutir, resultando en una mandíbula más estable [34]. La inserción del Forsus crea una reprogramación motora resultando en una adaptación postural que permite la respuesta de crecimiento [34].

Este tratamiento ha demostrado ser efectivo en la reducción del overjet en $5,5 \mathrm{~mm}$ con una mejoría en la relación molar de 3,4 mm. En cuanto el crecimiento sagital del maxilar se encontraron valores de $1,2 \mathrm{~mm}$ de Pt A a N y $-2,1^{\circ}$ para sNA. Mejorías en la posición sagital maxilo-mandibular en un rango de $-1,7^{\circ}$ para ANB y de $-2,5 \mathrm{~mm}$ de Wits [61]. Se muestran efectos dentales de $66 \%$ de la corrección sagital versus los esqueléticos dada por cambios dentoalveolares ya que el forsus no tiene mayores efectos esqueléticos [63]. Las relaciones oclusales se mejoraron por el movimiento distal de los molares maxilares y el movimiento mesial de los molares mandibulares, con retrusión de los incisivos superiores y la protrusión de los incisivos inferiores (reducción de 4,6 mm de overjet). La intrusión y protrusión de los incisivos inferiores reduce el overbite en $1,2 \mathrm{~mm}$ y el plano oclusal rota en $4,2^{\circ}$ abajo como resultado de la intrusión de los incisivos inferiores y los molares maxilares [63, 64].

Este aparato aplica una fuerza abajo y atrás de la mandíbula y causa una pequeña rotación posterior e incremento de la altura facial anterior inferior $[63,64]$. El forsus debe actuar por lo menos 6 meses para permitir una adecuada adaptación neuromuscular y permitir un resultado estable a largo plazo [66].

\section{Aparato de Reposicionamiento Anterior Mandibular (MARA)}

Es un aparato propuesto primero por Eckhart en 1998 como alternativa para el Herbst, con mayores ventajas al acortar la duración del tratamiento. Tiene un diseño rígido y no tiene conexión continua del arco superior al inferior [67]. Es fabricado en coronas de acero inoxidable apoyadas en los primeros molares permanentes maxilares y mandibulares (figuras 11 y 12). Ha sido usado a través de las etapas de dentición mixta y dentición permanente temprana.

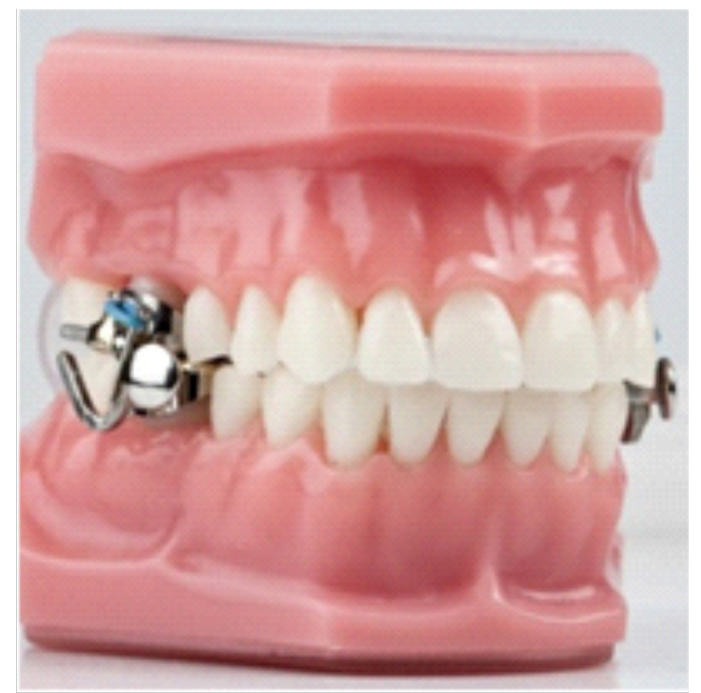

Figura 11. MARA en posición

Fuente: Catálogo Ormco, 2012 [71] 


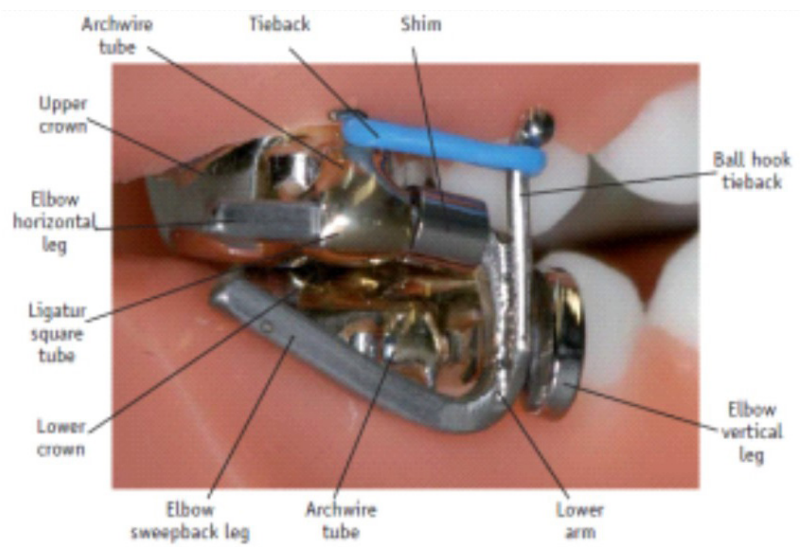

Figura 12. Señalización de cada uno de los componentes del MARA Fuente: Gonner, Ozkan, Jahn y Toll, 2007 [68]

El MARA es similar al Herbst porque ofrece efectos esqueléticos significativos (aumento de Co- $\mathrm{Gn}$ de $+2,0$ mm y disminución del maxilar A- perp NA de $-1,4$ mm después del seguimiento).

Los cambios dentoesqueléticos no son tan pronunciados con reducción de la proinclinación del incisivo inferior ( $2 \mathrm{~mm}$ a $3,3 \mathrm{~mm}$ cuando se compararon con los controles) y con una extrusión del primer molar inferior de 1,6 mm y mesialización de 1,2 mm [67, 69, 70]. El movimiento mesial de los anteriores inferiores resulta de una fuerza activa mesial (más en cuerpo que inclinación), con una proinclinación de 1,7 mm [68].

Con respecto al tiempo de tratamiento los estudios muestran que existe una mayor elongación mandibular en la etapa puberal (Co- $\mathrm{Gn}+2,6 \mathrm{~mm}$, con respecto a los pacientes controles), con cambios esqueléticos mandibulares y mínimas compensaciones dentoalveolares [72].

El MARA actúa mediante la restricción del paciente para cerrar en una relación natural de clase II, debido a que las extensiones interfieren haciendo que la mandíbula se tenga que posicionar adelante para que ocurra la intercuspidación.

Debido al diseño del MARA, es posible tener efectos indeseables en los movimientos dentales. En el plano sagital, se puede observar una rotación distal de los molares maxilares o una rotación mesial de los molares inferiores (esto se puede controlar con una barra traspalatina y un arco lingual). Verticalmente se puede observar frecuentemente una intrusión relativa de los molares debido a la compresión del espacio libre tras el retiro de las coronas de acero inoxidable; este fenómeno es corto y con frecuencia se autocorrige [73].

\section{AdvanSync}

El doctor Terry Dischinger diseñó el M2M para la corrección de la maloclusión clase II, el cual es una modificación del Herbst, con un tamaño más pequeño y cómodo para el paciente, sin necesidad de procesos de laboratorio. El aparato fue lanzado al mercado con el nombre de AdvanSync de casa comercial Ormco y hasta el momento no hay suficientes investigaciones en la literatura ortodóntica sobre sus efectos [68].

Es un aparato fijo diseñado para ser adherido a los molares superiores e inferiores que se mantendrá en posición durante 7 a 12 meses con periodos de ajuste, al mismo tiempo del tratamiento de ortodoncia (figura 13). La mandíbula se avanza hasta llevarla a una posición borde a borde y se reactiva entre $2 \mathrm{~mm}$ a $4 \mathrm{~mm}$ cada 12 semanas. Los brazos del AdvanSync tienen la mitad del tamaño de los de los aparatos Herbst tradicionales, lo que genera en una mayor comodidad para los pacientes, además se coloca más posterior y prácticamente no se ve.

A nivel esquelético se logra un cambio en SNB de $6,4^{\circ}$, en Anb de $4,2^{\circ}$, Wits $6,3 \mathrm{~mm}$ y de $6,7^{\circ}$ en $\mathrm{Na}$ - APog y un aumento significativo de $4,3^{\circ}$ en SN- GoGn y $3,1^{\circ}$ en MP- FH. Estudios recientes muestran un incremento en la longitud mandibular de $+1,4 \mathrm{~mm}$ [68]. Verticalemente muestra incremento en la rotación abajo y atrás del plano funcional oclusal, por la proinclinación significativa de los incisivos mandibulares [68].

A nivel dental se reporta lingualización del incisivo superior de $7,4^{\circ}$ y vestibularización del incisivo inferior (IMPA pasa de $98,5^{\circ}$ a $100,9^{\circ}$ ), con consecuente disminución del overjet de $7,2 \mathrm{~mm}$. La relación molar mejora en $5,3 \mathrm{~mm}$ y la longitud mandibular aumenta $3 \mathrm{~mm}$, mientras a nivel del maxilar superior se encuentra una restricción significativa de su crecimiento anteroposterior [68, 74]. Verticalmente se incrementan todas las dimensiones, por tanto estaría contraindicado en pacientes hiperdivergentes. Los efectos totales del aparato son un $66 \%$ de cambios esqueléticos y un $33 \%$ de cambios dentales.

$\mathrm{Al}$ realizar simultáneamente la terapia de AdvanSync con la ortodoncia correctiva, se controlan rápidamente los efectos secundarios a nivel de los molares (intrusiones) y a nivel de los incisivos superiores e inferiores [74]. Se recomienda que durante el tratamiento el paciente pase el pico de crecimiento para mayor estabilidad [68]. 


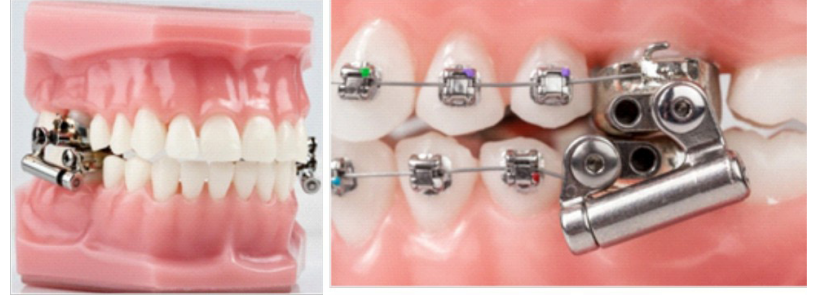

Figura 13. Aparato AdvanSync (M2M) anclado a los primeros molares maxilares y mandibulares

Fuente: Catálogo Ormco, 2012 [71]

\section{Estabilidad y recidiva}

Después del tratamiento con aparatos funcionales la recidiva es posible, y la retención es esencial. Los cambios definitivos sólo son posibles si todos los tejidos que son dependientes para mejorar la función de la articulación pueden ser influenciados con tratamiento ortodóntico u ortopédico. También existen otras influencias, especialmente la herencia que actúa durante el periodo de crecimiento o incluso después. El éxito del tratamiento con aparatos funcionales depende del tiempo en que se use el aparato, e incluso el tiempo en el que está trabajando en los dientes y maxilares [75].

En la efectividad del tratamiento es muy importante la medición del potencial de crecimiento, el momento en el que se coloque y el estadio de maduración en los pacientes, especialmente para los aparatos ortopédicos. En la tabla 3 se muestra el resumen de algunos aspectos de los diferentes aparatos funcionales y ortopédicos.

Tabla 3. Resumen de las diferencias entre los aparatos funcionales y ortopédicos

\begin{tabular}{|c|c|c|c|}
\hline Tipos de aparato & $\begin{array}{c}\text { Efectos esqueléticos, dentales y } \\
\text { musculares }\end{array}$ & $\begin{array}{l}\text { Momento del tratamiento } \\
\text { Duración del tratamiento }\end{array}$ & $\begin{array}{c}\text { Fuerza } \\
\text { estabilidad }\end{array}$ \\
\hline $\begin{array}{l}\text { Funcionales } \\
\text { Bionator }\end{array}$ & $\begin{array}{l}\text { - Aumento de la longitud mandibular. } \\
\text { - Aumento en la altura de la rama. } \\
\text { - Apertura del ángulo mandibular. } \\
\text { - Incremento en la altura facial antero } \\
\text { inferior. } \\
\text { - Retroinclinación de incisivos superiores. } \\
\text { - } \text { Proinclinación de incisivos inferiores. } \\
\text { - } \text { Corrección de relación molar. } \\
\text { peducción de la presión de los músculos } \\
\text { periorales. }\end{array}$ & $\begin{array}{l}\text { Su uso debe ser durante el } \\
\text { pico de crecimiento puberal. } \\
\text { El aparto se debe usar día y } \\
\text { noche con controles de } 3 \text { a } 5 \\
\text { semanas, con un promedio } \\
\text { de duración del tratamiento } \\
\text { de } 12 \text { meses. }\end{array}$ & $\begin{array}{l}\text { Utilizan la propia función de la } \\
\text { musculatura peridentaria, cuyo } \\
\text { equilibrio tratan de restablecer como } \\
\text { medio terapéutico para mover los } \\
\text { dientes. Son meros transmisores } \\
\text { de fuerzas que proceden de la } \\
\text { acción muscular y se convierten en } \\
\text { inductores de la acción. } \\
\text { Adecuada estabilidad tres años } \\
\text { después. }\end{array}$ \\
\hline Twin block & $\begin{array}{l}\text { - Aumento de la longitud mandibular. } \\
\text { - Incremento en la altura facial anterior y } \\
\text { posterior. } \\
\text { - Inhibición del crecimiento hacia adelante } \\
\text { del maxilar. } \\
\text { - Apertura del ángulo goniaco. } \\
\text { - Avance del mentón y pogonion. } \\
\text { - Reducción del ovejet. } \\
\text { - Protrusión y proinclinación de incisivos } \\
\text { - } \text { inferiores. } \\
\text { - } \text { Corroinclinación de superiores. } \\
\end{array}$ & $\begin{array}{l}\text { Su uso debe ser durante el } \\
\text { pico de crecimiento puberal. } \\
\text { El uso del aparato debe ser } \\
\text { de } 24 \text { horas durante } 9 \text { a } 12 \\
\text { meses para lograr los efectos } \\
\text { antes descritos. }\end{array}$ & \\
\hline Ortopédicos & $\begin{array}{l}\text { - Aumento en la longitud mandibular de } \\
\text { - Aumento de la altura facial anterior } \\
\text { inferior. } \\
\text { - Avance del mentón de } 2,5 \mathrm{~mm} \text {. } \\
\text { - Rotación posterior mandibular. } \\
\text { - Movimiento distal de los molares de los } \\
\text { - Retracción de los incisivos maxilares. } \\
\text { - Protrusión y proinclinación de incisivos } \\
\text { inferiores. }\end{array}$ & $\begin{array}{l}\text { El periodo ideal para el } \\
\text { tratamiento con Herbst es } \\
\text { en la dentición permanente } \\
\text { o justo después del pico de } \\
\text { crecimiento puberal. } \\
\text { El tiempo de tratamiento es } \\
\text { en promedio es de } 8 \text { meses, } \\
\text { seguido de una terapia de } \\
\text { ortodoncia interceptiva } \\
\text { correctiva, para completar un } \\
\text { tiempo total de tratamiento } \\
\text { de } 18 \text { meses }\end{array}$ & $\begin{array}{l}\text { Libera aproximadamente } 200-250 \text { grs } \\
\text { de fuerza durante el cierre. } \\
\text { Establidad a corto plazo. }\end{array}$ \\
\hline
\end{tabular}


(viene)

\begin{tabular}{|c|c|c|c|}
\hline Tipos de aparato & $\begin{array}{c}\text { Efectos esqueléticos, dentales y } \\
\text { musculares }\end{array}$ & $\begin{array}{l}\text { Momento del tratamiento } \\
\text { Duración del tratamiento }\end{array}$ & $\begin{array}{c}\text { Fuerza } \\
\text { estabilidad }\end{array}$ \\
\hline Jasper jumper & $\begin{array}{l}\text { - Restricción del crecimiento anterior del } \\
\text { maxilar. } \\
\text { - Protrusión mandibular. } \\
\text { - Movimiento distal de los molares } \\
\text { maxilares. } \\
\text { - Protracción dentoalveolar mandibular. } \\
\text { - Retroinclinación de incisivos superiores. }\end{array}$ & $\begin{array}{l}\text { Es ventajoso comenzar el } \\
\text { tratamiento en pacientes } \\
\text { adolescentes cuando la } \\
\text { mayoría de los dientes } \\
\text { permanentes han aparecido. } \\
\text { No se recomienda en } \\
\text { dentición mixta. } \\
6 \text { meses para nivelación y } \\
\text { preparación de anclaje, } 6 \\
\text { a } 9 \text { meses para el uso del } \\
\text { Jumper y } 12 \text { meses para la } \\
\text { finalización. }\end{array}$ & $\begin{array}{l}\text { Genera una fuerza aproximada } \\
60 \text { - } 250 \text { gr. Dejar el Jumper pasivo en } \\
\text { boca durante } 3 \text { a } 4 \text { meses aumenta la } \\
\text { estabilidad de los resultados. }\end{array}$ \\
\hline Forsus & $\begin{array}{l}\text { - Reducción del overjet. } \\
\text { - Movimiento distal de los molares } \\
\text { maxilares. } \\
\text { - Movimiento mesial e intrusivo de los } \\
\text { molares mandibulares. } \\
\text { - Retrusión de incisivos superiores. } \\
\text { Protrusión e intrusión de incisivos } \\
\text { inferiores. } \\
\text { - No tiene mayores efectos esqueléticos. } \\
\text { - Rotación abajo y atrás de la mandíbula } \\
\text { e incremento de la altura facial anterior } \\
\text { inferior. }\end{array}$ & $\begin{array}{l}\text { Pacientes en dentición } \\
\text { permanente. } \\
\text { El tiempo de tratamiento es } \\
\text { de } 8 \text { a } 12 \text { meses }\end{array}$ & $\begin{array}{l}\text { Produce aproximadamente } 150 \\
\text { a } 200 \text { gr de la fuerza cuando está } \\
\text { totalmente comprimido. } \\
\text { Buena estabilidad si se usa por lo } \\
\text { menos } 6 \text { meses para permitir la } \\
\text { reorganización neuromuscular. }\end{array}$ \\
\hline MARA & $\begin{array}{l}\text { - Proinclinación y protrusión de los } \\
\text { incisivos inferiores. } \\
\text { - Extrusión y mesialización de los molares } \\
\text { inferiores. } \\
\text { - Distalización de molares superiores. } \\
\text { - Reducción del overbite. } \\
\text { - Incrementos en la longitud mandibular, } \\
\text { con pocos efectos en el maxilar. }\end{array}$ & $\begin{array}{l}\text { Existe una mayor elongación } \\
\text { mandibular en la etapa } \\
\text { puberal. } \\
\text { Tiempo de tratamiento } 12 \\
\text { meses. }\end{array}$ & $\begin{array}{l}\text { Se demuestra una recidiva en los } \\
\text { incisivos inferiores para los pacientes } \\
\text { adolescentes de } 0,5 \mathrm{~mm} \text { y para los } \\
\text { adultos } 0,2 \mathrm{~mm} \text {, se nota mínimo } \\
\text { incremento en el overjet } 0,1 \mathrm{~mm} \text {. }\end{array}$ \\
\hline AdvanSync & $\begin{array}{l}\text { - Incremento en la longitud mandibular. } \\
\text { - Aumenta la rotación mandibular abajo } \\
\text { y atrás. } \\
\text { - Restricción de crecimiento } \\
\text { anteroposterior del maxilar superior. } \\
\text { - Proinclinación de incisivos inferiores. } \\
\text { - Retroinclinación de incisivos superiores. }\end{array}$ & $\begin{array}{l}\text { Durante el pico de } \\
\text { crecimiento. } \\
\text { Tiempo de tratamiento } 12 \\
\text { meses. }\end{array}$ & $\begin{array}{l}\text { Se recomienda que durante el } \\
\text { tratamiento el paciente pase el } \\
\text { pico de crecimiento para mayor } \\
\text { estabilidad. }\end{array}$ \\
\hline
\end{tabular}

Fuente: elaboración propia

\section{Conclusiones}

- Con el uso de los aparatos funcionales y ortopédicos se puede aumentar la longitud mandibular, restringir el crecimiento anteroposterior del maxilar, reducir el overjet, permitir la proinclinación de incisivos inferiores y retroinclinación de los incisivos superiores.

- Se debe tener precaución en los pacientes con crecimiento vertical, ya que el uso de estos aparatos tiende a aumentar la rotación mandibular abajo y atrás.

- Los estudios clínicos y experimentales han mostrado que hay mejor respuesta al crecimiento mandibular con aparatos funcionales cuando el tratamiento se inicia durante el periodo circumpuberal.

\section{Referencias}

[1] Kerr WJ, Miller S, Ayme B, Wilhelm N. Mandibular form and position in 10-year-old boys. Am J Orthod Dentofacial Orthop. 1994; 106(2): 115-20.

[2] Woodside DG. The present role of the general practitioner in orthodontics. Dent Clin North Am. 1968; 1: 483-508.

[3] Massler M, Frankel JM. Prevalence of malocclusion in children aged 14 to 18 years. Am J Orthod. 1951; 37(10): 751-68.

[4] McNamara JA, Jr. Components of class II malocclusion in children 8-10 years of age. Angle Orthod. 1981; 51(3): 177-202. 
[5] Graber TR, Petrovic T. A orthodontic and dentofacial orthopedic treatment. 5a. ed. Louis: Mosby; 2009.

[6] Bishara SE, Ziaja RR. Functional appliances: a review. Am J Orthod Dentofacial Orthop. 1989; 95(3): 250-8.

[7] Grabowski T. Principios de anatomía y fisiología. 7a ed. Chicago: Harcout; 1998.

[8] Netter FH. Atlas de anatomía humana. 4ª ed. Barcelona: Elsevier-Masson; 2007, 2009.

[9] Montenegro R. Factores que regulan la morfogénesis y el crecimiento mandibular humano. Int J Odontostomat. 2007; 1(1): 7-15.

[10] Bjork A. Craneal base development. Am J Orthod. 1955; 41(198): 198-225.

[11] Buschang PH, Gandini Junior LG. Mandibular skeletal growth and modelling between 10 and 15 years of age. Eur J Orthod. 2002; 24(1): 69-79.

[12] Gandini LG, Jr., Buschang PH. Maxillary and mandibular width changes studied using metallic implants. Am J Orthod Dentofacial Orthop. 2000; 117(1): 75-80.

[13] Lux CJ, Conradt C, Burden D, Komposch G. Transverse development of the craniofacial skeleton and dentition between 7 and 15 years of age. A longitudinal postero-anterior cephalometric study. Eur J Orthod. 2004; 26(1): 31-42.

[14] Buschang PH, Santos-Pinto A. Condylar growth and glenoid fossa displacement during childhood and adolescence. Am J Orthod Dentofacial Orthop. 1998; 113(4): 437-42.

[15] Enlow D. Essentials of facial growth. 2a. ed. Ann Arbor, MI: Needham Press; 2008.

[16] Enlow DH, Harris DB. A study of postnatal growth of the mandible. Am J Orthodont. 1964; 50: 25-50.

[17] Ruf S, Pancherz, H. When is the ideal period for herbst therapy- early or late? Semin Orthod. 2003; 9: 47-56.

[18] Lewis AB, Roche AF, Wagner B. Growth of the mandible during pubescence. Angle Orthod. 1982; 52(4): 325-42.

[19] Ochoa BK, Nanda RS. Comparison of maxillary and mandibular growth. Am J Orthod Dentofacial Orthop. 2004; 125(2): 148-59.

[20] Souza AM, Martinelli E. Mandibular growth during adolescence. Angle Orthodontist. 2006; 76(5): 1-9.

[21] Katsavrias EG, Halazonetis DJ. Condyle and fossa shape in Class II and Class III skeletal patterns: a morphometric tomographic study. Am J Orthod Dentofacial Orthop. 2005; 128(3): 337-46.

[22] Riesmeijer AM, Prahl-Andersen B, Mascarenhas AK, Joo BH, Vig KW. A comparison of craniofacial Class I and Class II growth patterns. Am J Orthod Dentofacial Orthop. 2004; 125(4): 463-71.
[23] Stahl F, Baccetti T, Franchi L, McNamara JA, Jr. Longitudinal growth changes in untreated subjects with Class II Division 1 malocclusion. Am J Orthod Dentofacial Orthop. 2008; 134(1): 125-37.

[24] Oudet C, Petrovic A, Stutzmann J. Time-dependent effects of a 'functional'-type orthopedic appliance on the rat mandible growth. Chronobiol Int. 1984; 1(1): 51-7.

[25] Petrovic AG, Stutzmann JJ, Oudet CL. Control processes in postnatal growth of mandibular condyle cartilage. Rev Iberoam Ortod. 1986; 6(1): 11-58.

[26] Bishara SE, Guardo CR. Ortodoncia. Ohio: McGrawHill; 2003.

[27] Araujo AM, Buschang PH, Melo AC. Adaptive condylar growth and mandibular remodelling changes with bionator therapy. An implant study. Eur J Orthod. 2004; 26(5): 515-22.

[28] Rabie AB, Zhao Z, Shen G, Hagg EU, Dr O, Robinson $\mathrm{W}$. Osteogenesis in the glenoid fossa in response to mandibular advancement. Am J Orthod Dentofacial Orthop. 2001; 119(4): 390-400.

[29] Rabie AB, Shum L, Chayanupatkul A. VEgF and bone formation in the glenoid fossa during forward mandibular positioning. Am J Orthod Dentofacial Orthop. 2002; 122(2): 202-9.

[30] Voudouris JC, Kuftinec MM. Improved clinical use of Twin-block and Herbst as a result of radiating viscoelastic tissue forces on the condyle and fossa in treatment and long-term retention: growth relativity. Am J Orthod Dentofacial Orthop. 2000; 117(3): 247-66.

[31] Canut BL. Ortodoncia clínica y terapéutica. 2a. ed. Barcelona: Masson; 2000.

[32] Whetten LL, Johnston LE, Jr. The control of condylar growth: an experimental evaluation of the role of the lateral pterygoid muscle. Am J Orthod. 1985; 88(3): 181-90.

[33] Graber TR, Petrovic, A. Ortopedia dentofacial con aparatos funcionales. 2a. ed. Buenos Aires: Panamericana; 1995.

[34] Sood S, Kharbanda OP, Duggal R, Sood M, Gulati S. Neuromuscular adaptations with flexible fixed functional appliance. A 2-year follow-up study. J Orofac Orthop. 2011; 72(6): 434-45.

[35] Marsico E, Gatto E, Burrascano M, Matarese G, Cordasco G. Effectiveness of orthodontic treatment with functional appliances on mandibular growth in the short term. Am J Orthod Dentofacial Orthop. 1999; 139(1): 24-36.

[36] Baccetti T, Franchi L, Toth LR, McNamara JA, Jr. Treatment timing for Twin-block therapy. Am J Orthod Dentofacial Orthop. 2000; 118(2): 159-70.

[37] Franchi L, Baccetti T. Prediction of individual mandibular changes induced by functional jaw orthopedics followed by fixed appliances in Class II patients. Angle Orthod. 2006; 76(6): 950-4. 
[38] Cozza P, Baccetti T, Franchi L, De Toffol L, McNamara JA, Jr. Mandibular changes produced by functional appliances in Class II malocclusion: a systematic review. Am J Orthod Dentofacial Orthop. 2006; 129(5): 599 e112; discussion e1-6.

[39] Mills CM, McCulloch KJ. Treatment effects of the twin block appliance: a cephalometric study. Am J Orthod Dentofacial Orthop. 1998; 114(1): 15-24.

[40] Trenouth MJ. Cephalometric evaluation of the Twinblock appliance in the treatment of Class II Division 1 malocclusion with matched normative growth data. Am J Orthod Dentofacial Orthop. 2000; 117(1): 54-9.

[41] Almeida MR, Henriques JF, Almeida RR, Almeida-Pedrin RR, Ursi W. Treatment effects produced by the Bionator appliance. Comparison with an untreated Class II sample. Eur J Orthod. 2004; 26(1): 65-72.

[42] Faltin KJ, Faltin RM, Baccetti T, Franchi L, Ghiozzi B, McNamara JA, Jr. Long-term effectiveness and treatment timing for Bionator therapy. Angle Orthod. 2003; 73(3): 221-30.

[43] Toth LR, McNamara JA, Jr. Treatment effects produced by the twin-block appliance and the FR-2 appliance of Frankel compared with an untreated Class II sample. Am J Orthod Dentofacial Orthop. 1999; 116(6): 597-609.

[44] Catálogo Dentaurum. 2013. http://www.dentaurum. de/files/989-965-20.pdf

[45] Pancherz H. The Herbst appliance. Its biologic effects and clinical use. Am J Orthod. 1985; 87(1): 1-20.

[46] Pancherz $\mathrm{H}$. The effect of continuous bite jumping on the dentofacial complex: a follow-up study after Herbst appliance treatment of class II malocclusions. Eur J Orthod. 1981; 3(1): 49-60.

[47] Pancherz H, Ruf S. The herbst appliance: research-based clinical management. Chicago: Quintessence, 2008.

[48] Serbesis-Tsarudis C, Pancherz H. "Effective" тмJ and chin position changes in Class II treatment. Angle Orthod. 2008; 78(5): 813-8.

[49] De Almeida MR, Henriques JF, de Almeida RR, Weber U, McNamara JA, Jr. Short-term treatment effects produced by the Herbst appliance in the mixed dentition. Angle Orthod. 2005; 75(4): 540-7.

[50] Pancherz H, Fischer S. Amount and direction of temporomandibular joint growth changes in Herbst treatment: a cephalometric long-term investigation. Angle Orthod. 2003; 73(5): 493-501.

[51] Ruf S, Pancherz H. Temporomandibular joint remodeling in adolescents and young adults during Herbst treatment: A prospective longitudinal magnetic resonance imaging and cephalometric radiographic investigation. Am J Orthod Dentofacial Orthop. 1999; 115(6): 607-18.
[52] Pancherz H. Vertical dentofacial changes during Herbst appliance treatment. A cephalometric investigation. Swed Dent J Suppl. 1982; 15: 189-96.

[53] Martin J, Pancherz H. Mandibular incisor position changes in relation to amount of bite jumping during Herbst/multibracket appliance treatment: a radiographic-cephalometric study. Am J Orthod Dentofacial Orthop. 2009; 136(1): 44-51.

[54] Hansen K, Koutsonas TG, Pancherz H. Long-term effects of Herbst treatment on the mandibular incisor segment: a cephalometric and biometric investigation. Am J Orthod Dentofacial Orthop. 1997; 112(1): 92-103.

[55] Herrera FS, Henriques JF, Janson G, Francisconi MF, de Freitas KM. Cephalometric evaluation in different phases of Jasper jumper therapy. Am J Orthod Dentofacial Orthop. 2011; 140(2): e77-84.

[56] Karacaya SA. Forsus nitinol flat spring and jasper jumper corrections of class II division 1 malocclusions. Angle Orthod. 2006; 76: 666-72.

[57] Korrodi A. Los aparatos funcionales fijos, una clasificación actualizada. The Orthodontic Ciberjournal. 2001 june.http://orthocj.com/2001/06/los-aparatos-funcionales-fijos-una-clasificacin-actualizada/

[58] American orthodontics. Complete AO product catalog en: www. americanortho.com/downloads/catalog/AOcatalog-2011.pdf.

[59] Nalbantgila DA. Skeletal, dental and soft-tissue changes induced by the jasper jumper appliance in late adolescence. Angle Orthod. 2005; 75: 426-36.

[60] Kucukkeles N, Ilhan I, Orgun IA. Treatment efficiency in skeletal Class II patients treated with the jasper jumper. Angle Orthod. 2007; 77(3): 449-56.

[61] Franchia L. Effectiveness of comprehensive fixed appliance treatment used with the forsus fatigue resistant device in class II patients. Angle Orthod. 2011; 81: 678-83.

[62] Vogt W. The forsus fatigue device. JCo. 2006; 40(6): 36877; quiz 358.

[63] Gunay EA, Arun T, Nalbantgil D. Evaluation of the Immediate Dentofacial Changes in Late Adolescent Patients Treated with the Forsus FRD. Eur J Dent. 2011; 5(4): 423-32.

[64] Heinig N, Goz G. Clinical application and effects of the Forsus spring. A study of a new Herbst hybrid. J Orofac Orthop. 2001; 62(6): 436-50.

[65] 3M Unitek Catalog. www.3Munitekcatalog.Multimedia $3 \mathrm{~m} . \mathrm{com}$

[66] Sood S, Kharbanda OP, Duggal R, Sood M, Gulati S. Muscle response during treatment of Class II Division 1 malocclusion with Forsus Fatigue Resistant Device. J Clin Pediatr Dent. 2011; 35(3): 331-8. 
[67] Ghislanzoni LT, Toll DE, Defraia E, Baccetti T, Franchi L. Treatment and posttreatment outcomes induced by the Mandibular Advancement Repositioning Appliance; a controlled clinical study. Angle Orthod. 2011; 81(4): 684-91.

[68] Gonner U, Ozkan V, Jahn E, Toll DE. Effect of the MARA appliance on the position of the lower anteriors in children, adolescents and adults with Class II malocclusion. J Orofac Orthop. 2007; 68(5): 397-412.

[69] Al-Jewair TS, Preston CB, Moll EM, Dischinger T. A comparison of the MARA and the AdvanSync functional appliances in the treatment of Class II malocclusion. Angle Orthod. 2012; 82(5): 907-14.

[70] Pangrazio MN, Pangrazio-Kulbersh V, Berger JL, Bayirli B. Treatment effects of the mandibular anterior repositioning appliance in patients with Class II skeletal malocclusions. Angle Orthod Mar 21.Epub ahead of print.
[71] Ormco Catalog. http://www.ormco.com/downloads/or mco-productcatalog.pdf

[72] Huanca Ghislanzoni LT, Baccetti T, Toll D, Defraia E, McNamara JA, Jr., Franchi L. Treatment timing of MARA and fixed appliance therapy of Class II malocclusion. Eur J Orthod Mar 15. Epub ahead of print.

[73] Kulbersh VB. Tretment effects of the mandibular anterior repositioning appliance on patients with class II malocclusion. Am J Orthod Dentofacial Orthoped. 2003; 123: 286-95.

[74] Perdomo AO. Cambios cefalométricos producidos por el aparato de avance mandibular, AdvanSync, en maloclusiones clase II. En proceso de publicación.

[75] Gottfried P. Considerations of functional aspects in dentofacial orthopedics andorthodontics: Sheldon Friel Memorial lecture. Am J Orthod Dentofacial Orthop. 1999; 115(4): 373-81. 
